\title{
Kondensation von Tiglinaldehyd mit Aceton
} von

\section{Dr. Fritz Dautwitz.}

Aus dem chemischen Laboratorium des Hofrates Prof. Ad. Lieben an der k. k. Universität in Wien.

(Vorgelegt in der Sitzung am 5. Juli 1906.)

Zweck der vorliegenden Untersuchung war, durch Kondensation von Tiglinaldehyd mit Aceton ein ungesättigtes Keton darzustellen von nachstehender Konstitutionsformel:

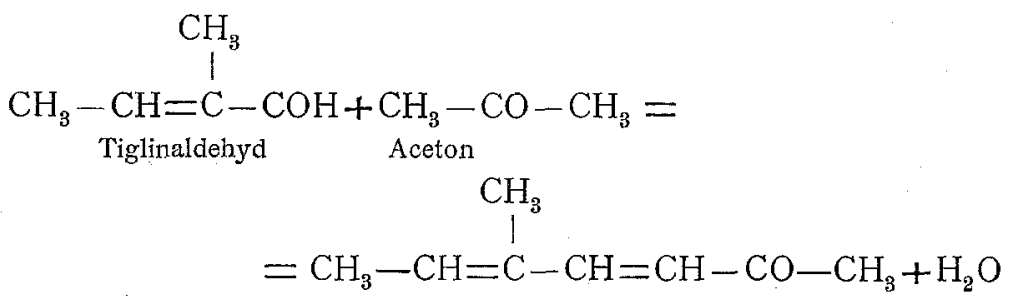

Der Tiglinaldehyd wurde nach dem Verfahren, das Li eben und Zeise 1 in ihrer Arbeit: "Kondensationsprodukte der Aldehyde und ihre Derivate", Monatshefte 7, p. 54, angegeben haben, durch Erhitzen eines Gemisches von Acet- und Propionaldehyd mit Natriumacetatlösung gewonnen. Die Synthese des Ketons aus dem Tiglinaldehyd und Aceton führte ich nun in nachstehender Weise aus.

Ein Gemisch von $75 \mathrm{~g}$ Tiglinaldehyd, $120 \mathrm{~g}$ Aceton und $300 \mathrm{~cm}^{3}$ Wasser wurde mit $20 \mathrm{~cm}^{3}$ einer zehnprozentigen $\mathrm{NaOH}$-Lösung versetzt und vierzig Stunden lang in der Schüttelmaschine bei einer Temperatur von 5 bis $10^{\circ} \mathrm{C}$. geschüttelt. Das Reaktionsprodukt, welches eine schwach bräunlich gefärbte Flüssigkeit darstellt, wurde von der wässerigen Schicht getrennt, letztere mit Kohlensäure gesättigt und der 
Destillation unterworfen. Das im Destillat sich abscheidende Öl wurde mit dem ersteren vereinigt. Das auf diese Weise erhaltene Rohprodukt wurde nun mit entwässertem Natriumsulfat getrocknet und hierauf im Vakuum bei 12 bis $14 \mathrm{~mm}$ Druck der fraktionierten Destillation unterworfen. Ich erhielt drei Fraktionen: etwa $10 \mathrm{~g}$ einer bei $91^{\circ} \mathrm{C}$. siedenden Flüssigkeit, eine zweite, welche die Hauptmenge bildet, $41 \mathrm{~g}$ ging bei $12 \mathrm{~mm}$ Druck fast konstant bei 92 bis $93^{\circ} \mathrm{C}$. über; der Rest, etwa $5 g$, hatte einen höheren Siedepunkt. Im Destillationskolben blieb eine beträchtliche Menge einer harzartigen, braunen Masse zurück, auf deren Untersuchung ich, da sie sich weder destillieren noch aus verschiedenen Lösungsmitteln in kristallinische Form bringen ließ, verzichten mußte.

Die Hauptfraktion stellt nach nochmaligem Destillieren im luftverdünnten Raume eine farblose, stark lichtbrechende, eigentümlich aromatisch riechende, leicht bewegliche Flüssigkeit dar, welche im Licht, namentlich bei Luftzutritt sich in kurzer Zeit in eine braungelb gefärbte harzartige Masse verwandelt. Bei der Destillation unter gewöhnlichem Druck zersetzt sich die Substanz. Für die Analyse und Dampfdichtebestimmung wurde das im Vakuum frisch destillierte Produkt verwendet.

I. $0.2529 \mathrm{~g}$ Substanz lieferten bei der Verbrennung $0.7126 \mathrm{~g}$ $\mathrm{CO}_{2}$ und $0.2199 \mathrm{~g} \mathrm{H}_{2} \mathrm{O}$;

II. $0 \cdot 1439 g$ Substanz lieferten bei der Verbrennung $0 \cdot 4082 \mathrm{~g}$ $\mathrm{CO}_{2}$ und $\mathrm{O} \cdot 1201 \mathrm{~g} \mathrm{H}_{2} \mathrm{O}$; daraus ergeben sich in 100 Teilen Substanz:

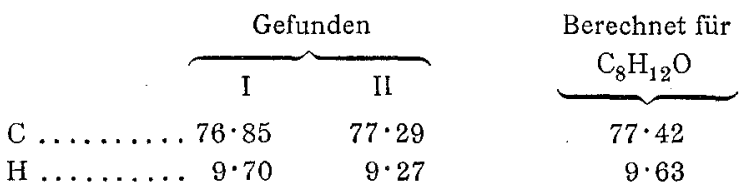

Die Molekulargröße der Substanz wurde durch Dampfdichtebestimmung nach Bleier-Kohn ermittelt.

I. $0 \cdot 0252 \mathrm{~g}$ Substanz erzeugten einen Überdruck von $19 \cdot 7 \mathrm{~mm}$.

II. $0.0344 \mathrm{~g}$ Substanz erzeugten einen Überdruck von $28.0 \mathrm{~mm}$.

Als Konstante, bezogen auf Naphthalin, gilt für beide Bestimmungen $975 \cdot 5$. 
Daraus ergeben sich nun folgende Werte:
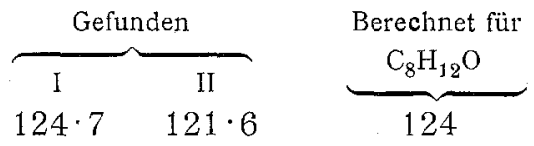

Das Keton zieht an der Luft sehr rasch Sauerstoff an; daher gaben Analysen des Körpers, wenn er einige Zeit in nicht sehr sorgfältig verschlossenen Gefäßen aufbewahrt wurde, bei der Verbrennung schlecht stimmende Werte (es wurde immer zu wenig $\mathrm{C}$ und $\mathrm{H}$ gefunden).

Das Keton addiert sehr leicht Brom. Ein quantitativer Bromadditionsversuch zeigte, daß der Körper, wie aus der oben angeführten Strukturformel $\mathrm{zu}$ ersehen ist, zwei doppelte Bindungen enthält und daher vier Atome Brom addiert.

$0.6654 \mathrm{~g}$ Substanz, in Schwefelkohlenstoff gelöst, verbrauchen $1 \cdot 70585 \mathrm{~g} \mathrm{Br}$, während die Theorie $1 \cdot 717 \mathrm{~g} \mathrm{Br}$ verlangt. Das Bromadditionsprodukt wurde nicht weiter untersucht, da es sich nach Abdunsten des Lösungsmittels unter Entwicklung von Bromwasserstoff in eine schwarze Masse verwandelt.

\section{Oxim des Ketons.}

Zur Darstellung des Oxims wurden $12 \mathrm{~g}$ Keton mit $8 \mathrm{~g}$ Hydroxylaminchlorhydrat und $50 \mathrm{~cm}^{3}$ zehnprozentiger Natronlauge versetzt und das Gemisch unter häufigem Umschütteln durch 24 Stunden sich selbst überlassen. Das Reaktionsgemisch wurde zuerst mit Kohlensäure gesättigt, hierauf mit Äther extrahiert, die ätherische Lösung über Pottasche getrocknet und der Äther abdestilliert. Es blieb ein dickflüssiges, fast farbloses, stark lichtbrechendes Öl zurück, welches bei der Destillation im Vakuum bei einem Druck von $13 \mathrm{~mm}$ zwischen 140 bis $141^{\circ} \mathrm{C}$. konstant überging. Das Destillat stellt eine farblose, dicke, stark lichtbrechende Flüssigkeit dar von eigentümlich aromatischem Geruch; sie erstarrt nach kurzer Zeit zu einer kristallinischen weißen Masse. Behufs Reinigung wurde aus Petroläther umkristallisiert. Das so erhaltene Oxim schmilzt bei $71^{\circ} \mathrm{C}$., die Analyse desselben lieferte folgendes Resultat: 
$0.1934 g$ Substanz lieferten bei der Verbrennungsanalyse $0 \cdot 4865 \mathrm{~g} \mathrm{CO}_{2}$ und $0.1593 \mathrm{~g} \mathrm{H}_{2} \mathrm{O}$.

$0 \cdot 4919 \mathrm{~g}$ Substanz lieferten bei der Stickstoffbestimmung nach Kjeldahl so viel Ammoniak, daß zur Neutralisation des letzteren $34.1 \mathrm{~cm}^{3} 1 / 10$ normale Säure benötigt wurden; dies entspricht $0.0477 \mathrm{~g}$ Stickstoff.

In 100 Teilen der Substanz:

\begin{tabular}{|c|c|c|}
\hline & Gefunden & $\begin{array}{l}\text { Berechnet für } \\
\mathrm{C}_{8} \mathrm{H}_{18} \mathrm{NO}\end{array}$ \\
\hline $\mathrm{C}$ & $68 \cdot 61$ & $69 \cdot 06$ \\
\hline$\ldots$ & $9 \cdot 15$ & $9 \cdot 35$ \\
\hline 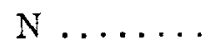 & $9 \cdot 75$ & $10 \cdot 07$ \\
\hline
\end{tabular}

Aus dem beschriebenen Keton und aus seinem Oxim hoffte ich durch Ringschluß zu einem Dimethylbenzol, respektive Trimethylpyridin zu gelangen:

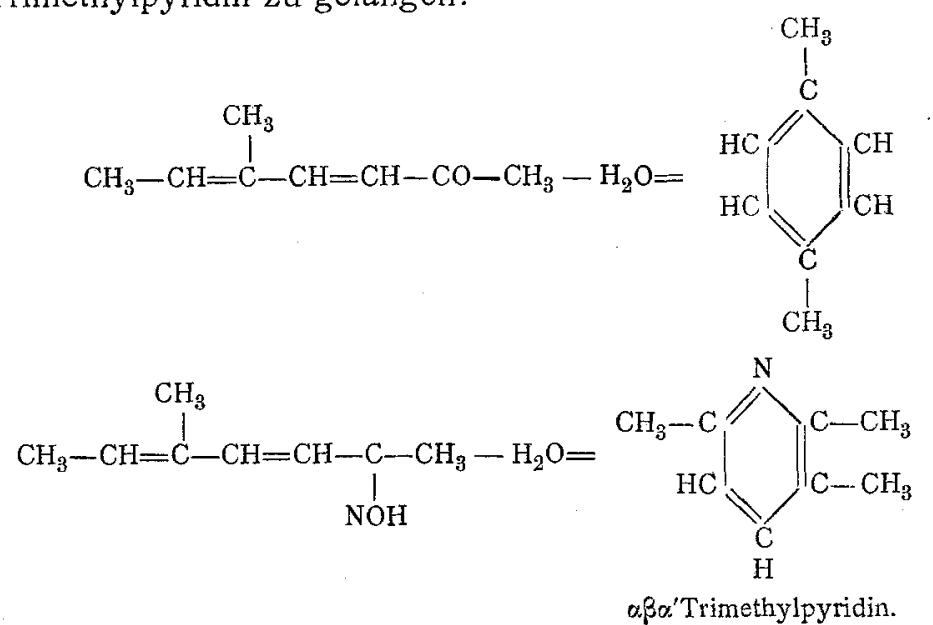

Trotz wiederholter Versuche mit Anwendung wasserentziehender Agenzien gelang es mir nicht, diese beiden Körper darzustellen.

Es erübrigt mir zum Schlusse die angenehme Pflicht, Herrn Prof. Dr. C. Pomeranz, unter dessen Leitung ich die vorstehenden Untersuchungen ausgeführt habe, meinen wärmsten und verbindlichsten Dank abzustatten. 